\title{
Ladungssicherung - ein aktuelles Thema
}

\author{
RALF WUNDERLICH, \\ GERRIT HASSELMANN \\ FRAUNHOFER-INSTITUT FÜR MATERIALFLUSS UND LOGISTIK IML, DORTMUND
}

Ladungssicherung ist derzeit ein aktuelles Thema mit einer hohen Brisanz. Schon kleine Fehler in diesem Bereich können große Schäden auslösen. Verstärkte Forschungsaktivitäten führen zu neuen Erkenntnissen, die in zukünftigen Maßnahmen Berücksichtigung finden sollten. Dies trifft auch für die Ladungssicherung von Betonfertigteilen zu.

Load securing presently is a very important issue. Small acts of negligence may lead to great damage. Recent research activities revealed new facts which should be considered when future measures are taken. This also concerns the securing of precast concrete elements.

\section{Ausgangssituation}

Bei dem Neubau von Gebäuden kommen in großem Umfang Fertigteile aus Beton zum Einsatz. Diese Fertigteile, wie z.B. Elementwände und -decken mit Gitterträgern, werden i. d. R. nach Kundenwunsch auf Maß hergestellt. Aufgrund dieser kundenspezifischen Anfertigung haben z.B. die Elementdecken zwar ähnliche geometrische Formen, aber fast immer unterschiedliche Abmessungen und Gewichte.

So müssen diese Elementdecken so geplant und gefertigt werden, dass sie auf der Baustelle mit einem Kran in einer festgelegten Reihenfolge auf dem Rohbau verlegt werden können. Dazu werden in den Betonwerken die Deckenelemente nach der Fertigung in der entsprechenden Reihenfolge in einem Block gestapelt. Als Unterlage für einen solchen Decken-Stapel werden entweder stabile Stahlrahmen oder Holzbalken verwendet. Zwischen die einzelnen Deckenelemente eines Stapels werden z.T. keine Zwischenlagen gelegt. Dabei liegt das obere Element mit seiner glatten Betonseite auf dem Gitterträger des unteren Elementes auf oder es werden Holzlatten zwischen die Betonseite des oberen Elementes und dem Gitterträger des unteren Elementes gelegt. Innerhalb eines solchen Stapels können aufgrund der vorgegebenen Verlegereihenfolge unterschiedlich große Decken liegen. Die Lagerung, Verladung und der Transport erfolgt in aller Regel ohne Schutz der Deckenelemente vor klimatischen Einflüssen, wie Regen, Schnee oder Frost.

Für den Transport zur Baustelle werden die Decken-Stapel auf offene LKW verladen. So liegen auf einem Sattelauflieger bis zu zwei Stapel, die jeweils 10 - 12 Tonnen schwer sein können. Die Decken-Stapel werden dann mit Hilfe von Zurrgurten und Zurrseilen durch Niederzurren auf den Ladeflächen gesichert.

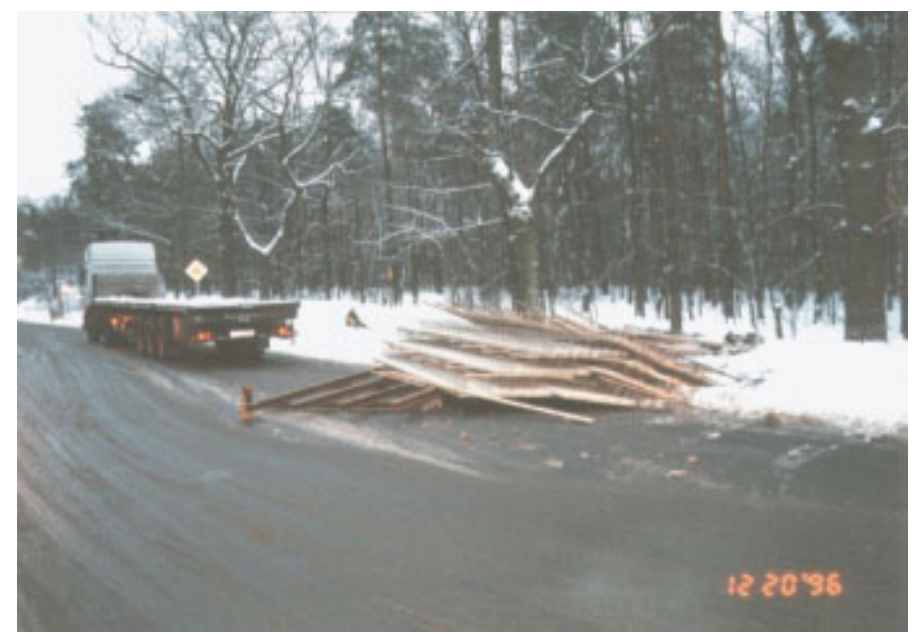

Abbildung 1: Die Folgen schlechter Ladungssicherung 


\section{Aktivitäten des Bundesverbandes Deutsche Beton- und Fertigteilindustrie (BDB)}

Die Fachgruppe Betonbauteile mit Gitterträgern im BDB beschäftigt sich schon seit geraumer Zeit mit der Ladungssicherung von Fertigteilen aus Beton auf Lkw. In Zusammenarbeit mit Fachleuten verschiedener Institutionen, wie u. a. dem Fraunhofer-Institut für Materialfluss und Logistik sowie betroffenen Firmen sollen Hilfestellungen für Fahrer und Verlader erarbeitet werden.

Bereits zu Beginn der Beratungen war klar, dass generell davon ausgegangen wird, dass aufgrund der spezifischen Eigenschaften der Fertigteile und der Art des Transportes nur eine kraftschlüssige Verladung, überwiegend durch Niederzurren, in Frage kommt.

Das entscheidende Kriterium zur Festlegung der erforderlichen Ladungssicherungsmittel bei der Sicherung durch Niederzurren sind die Reibwerte. Dazu wird auf die Reibwerte in der VDI-Richtlinie 2700 zurückgegriffen. Als Reibwerte werden dort 0,3 - 0,6 für den trockenen Zustand und 0,3 - 0,5 für den nassen Zustand angegeben.

Aufgrund der großen Streuung der angegebenen Reibwerte für Beton und der Vorgabe, im Zweifelsfall stets den kleinsten Reibwert anzunehmen, muss in der Regel der niedrigste Reibwert für die Auslegung bzw. Beurteilung der Ladungssicherungsmaßnahmen eingesetzt werden. Dies kann dazu führen, dass in Kontrollen sehr aufwändige Ladungssicherungsmaßnahmen mit einer im Extremfall unrealistisch hohen Anzahl von Zurrgurten gefordert werden.

Bezüglich der in der VDI-Richtlinie 2700 angegebenen Reibwerte bestand in der Fachgruppe die Frage, inwieweit diese Werte zutreffen und ob sie auf andere Materialpaarungen übertragen werden können. Aus diesem Grund sollten Reibwerte von verschiedenen Materialpaarungen (Beton, Holz, Stahl, etc.) auf realen Ladeflächen ermittelt werden. Der BDB beauftragte in einer ersten Versuchsreihe den TÜV Rheinland und das Fraunhofer-Institut IML, gemeinsam Zugversuche zur Ermittlung von Reibwerten durchzuführen. Dazu wurden die jeweils vom TÜV und vom Fraunhofer-IML entwickelten transportablen Messaufbauten verwendet. Diese Messaufbauten gestatten es, reale zu transportierende Güter in Bezug auf ihre Reibungseigenschaften auf realen Fahrzeugladeflächen zu untersuchen.

\section{Theorie der an Ladungen wirkenden Kräfte und deren Effekte}

Neben der Erdbeschleunigung treten auf den Ladeflächen von Lkw zusätzliche horizontale und vertikale Beschleunigungen auf. Die horizontalen Kräfte sind diejenigen, welche eine Ladung zum Rutschen bringen können.

Abhängig von den horizontalen Kräften, die bei einer Kurvenfahrt als Zentrifugalkräfte (Fliehkräfte) oder Bremsvorgängen des Fahrzeuges als Bremsverzögerungen auftreten, und den Reibwerten (Haft- und Gleitreibung) sowie der Geometrie der Ladung, beginnt das Gut zu rutschen.

Horizontal wirkenden Kräften entgegen steht eine Haftkraft, die bedingt ist durch die Reibung zwischen der Ladung und der Ladefläche. Überschreitet die horizontale Kraft die Haftkraft / -reibung, so beginnt die Ladung sich zu bewegen, sie gleitet.

Es werden zwei Arten der Reibung unterschieden und Kennwerte dazu als Koeffizienten angegeben.

1. Haftreibung: Schwellenwert, der zu Beginn einer Gleitbewegung zu überwinden ist, aber nicht ausreicht, um eine Relativbewegung zu erzeugen. Es wird die Haftreibungszahl $\mu_{0}$ als Koeffizient aus der Gewichtskraft $F_{G}$, die senkrecht zur Reibungsfläche wirkt, und der Haftreibungskraft $F_{S}$, die notwendig, ist die Haftreibung zu überwinden, errechnet:

$$
\mu_{0}=\mathrm{F}_{\mathrm{S}} / \mathrm{F}_{\mathrm{G}}
$$

2. Gleitreibung: Reibung, die direkt nach Überwindung der Haftreibung bei einer vorgegebenen Gleitgeschwindigkeit noch wirksam bleibt. Es wird die Gleitreibungszahl $\mu$ als Koeffizient aus der Gewichtskraft $F_{G}$, die senkrecht zur Reibungsfläche wirkt und der Gleitreibungskraft $F_{D}$, die notwendig ist die Gleitreibung zu überwinden, errechnet:

$$
\mu=F_{D} / F_{G}
$$

Werden horizontal auftretende Beschleunigungen, wie in der VDI-Richtlinie 2700 geschehen, in Vielfachen bzw. Bruchteilen der Erdbeschleunigung angegeben (0,8 g in Fahrtrichtung, 0,5 g quer und gegen Fahrtrichtung), so können diese Werte direkt mit den Reibwerten verglichen werden. 
Die Bedingungen lauten also, dass der kleinere der beiden Reibwerte (Haft- und Gleitreibung) größer als 0,8 bzw. 0,5 sein muss, wenn die Ladung ohne weitere Sicherung geladen werden soll. Die Ladung kann auch durch Erhöhung der Gewichtskraft $F_{G}$, z.B. durch Niederzurren und/oder eine formschlüssige Verladung, z.B. die Ladung stützt sich an den Bordwänden ab und/oder einer kraftschlüssigen Verladung durch Schräg- oder Diagonalzurren, gesichert werden.

\section{Ermittlung der Haft- und Gleitreibwerte in der Praxis}

Die Untersuchungen wurden mit offenen Sattelaufliegern und handelsüblichen Ladeflächen durchgeführt. Eine Ausnahme bildete ein spezieller Sattelauflieger, der mit einer fest aufgebrachten, speziellen Granulatgummierung versehen war. Bei den unterschiedlichen Sattelaufliegern handelte es sich um Fahrzeuge mit den im folgenden beschriebenen Ladeflächen:

- Siebdruckplatten, jeweils neuwertig und gebraucht

- Holzplanken gebraucht

- Holzplanken mit hervorstehenden Stahlteilen

- Siebdruckplatten mit spezieller Antirutsch-Gummierung

Auf diesen Ladeflächen wurden die Zugversuche zur Ermittlung der Reibwerte mit folgenden Fertigteilen bzw. Hilfsmitteln durchgeführt:

- Elementdeckenplatten

- Elementwandplatten

- Transportgestell

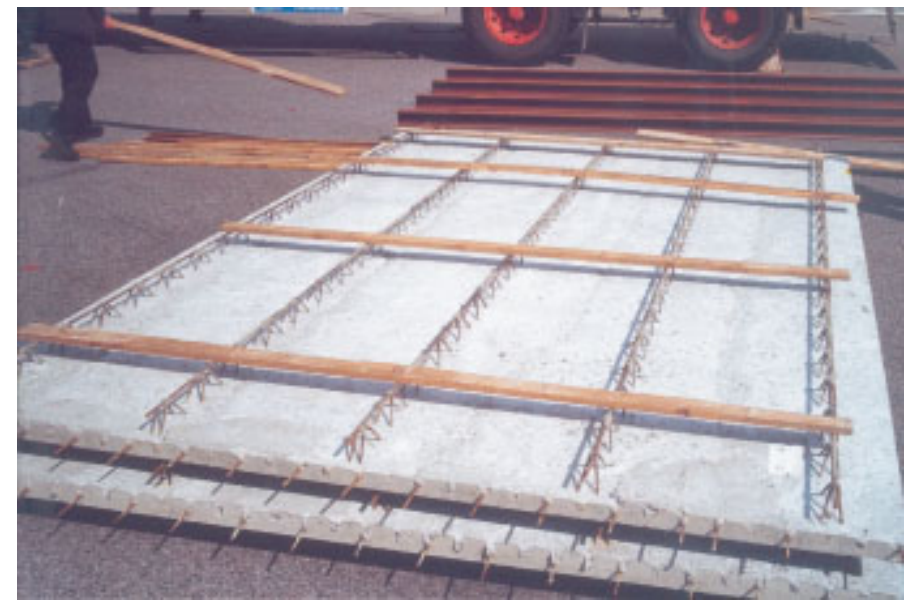

Abbildung 2: Elementdeckenplatten aus Beton mit Holzzwischenlage

Für die Prüfungen erfolgte die Verladung in Anlehnung an die üblichen Verfahren:

- Verladung auf einem Transportgestell aus Stahl mit und ohne Holzzwischenlagen

- Verladung direkt auf der Ladefläche, jeweils mit und ohne Holzzwischenlagen

- Verladung mit Antirutschmatten lose zwischengelegt 


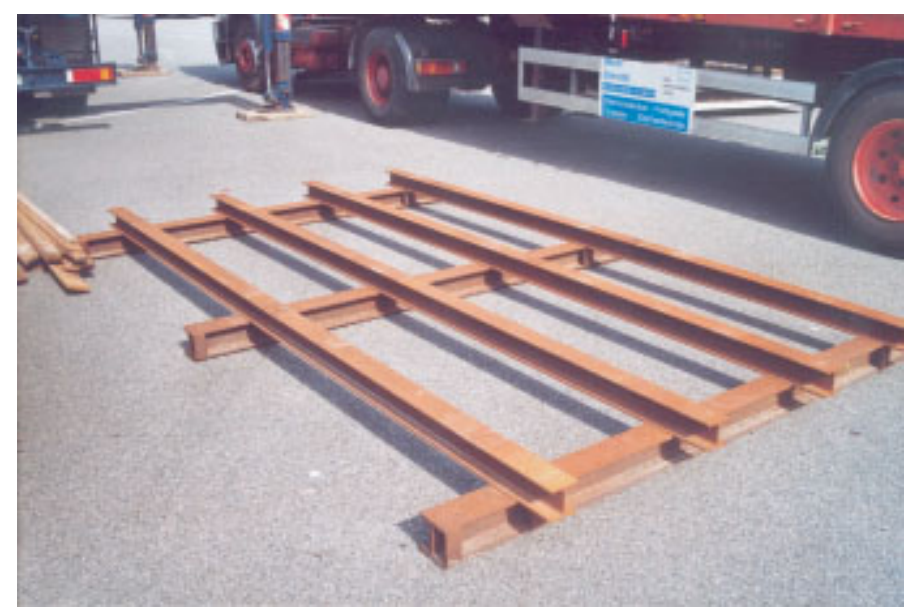

Abbildung 3: Transportgestell aus Stahl

\section{Ergebnisse der ersten Versuchsreihe}

Insgesamt lagen die Reibwerte im oberen Bereich der Toleranz, in Bezug auf die in der Reibwerttabelle der VDI-Richtlinie 2700 für Beton. Allerdings wird diese nur für die Materialpaarung gegen Holz angegeben. Einige der Materialpaarungen haben darüber hinaus noch deutlich höhere Reibwerte ergeben. Diese hohen Reibwerte würden bei der Auslegung der notwendigen Maßnahmen zur Ladungssicherung zu einer deutlichen Reduktion des Aufwandes führen.

Um die Ergebnisse in Fahrversuchen zu bestätigen bzw. noch zu vertiefen, wurde vom BDB beschlossen, auf dem Testgelände der DEKRA Automobil AG in Neumünster Bremsversuche mit einer Ladung von Betonfertigteilen durchzuführen.

Der Versuchsaufbau und die Bremsversuche sollten auf die laut VDI-Richtlinie 2700 maximal zu erwartende Bremsbeschleunigung von $\mathrm{a}=0,8 * \mathrm{~g}$ in Fahrtrichtung ausgelegt werden.

\section{Weitere Zugversuche und Bremsversuche im DEKRA-Crash- zentrum in Neumünster}

Für die im DEKRA-Crashzentrum in Neumünster stattgefundenen Zug- und Bremsversuche wurden zwei Verladeformen aus der Praxis der Verladung von Betonfertigteilen ausgewählt. Dabei handelte es sich um:

\section{Verladung von Elementwandplatten:}

Die untere Wandplatte lag dabei auf einem Transportgestell aus Stahl, mit Holzlatten (ca. $10 \mathrm{~cm}$ breit) als Zwischenlage auf. Der weitere Aufbau des Stapels erfolgte Wand auf Wand (Beton auf Beton) ohne weitere Zwischenlagen. Der gesamte Stapel von insgesamt vier Elementwänden und dem Stahlrahmen hatte ein Gewicht von $8.576 \mathrm{~kg}$.

\section{Verladung von Elementdeckenplatten:}

Die untere Deckenplatte lag mit einer Zwischenlage aus vier Holzbalken (ca. 10 x 15 cm) auf der Ladefläche. Der weitere Aufbau des Stapels erfolgte Decke auf Decke mit Zwischenlagen aus ca. $10 \mathrm{~cm}$ breiten Holzlatten (Beton/Holz/Stahlarmierung). Der gesamte Stapel von insgesamt sechs Elementdecken und Zwischenlagen hatte ein Gewicht von ca. 7.500 kg.

\section{Fahrzeug:}

Als Fahrzeug wurde ein offener Sattelauflieger mit einem „alten“ Siebdruckboden als handelsübliche Ladefläche eingesetzt. Dieser Auflieger wurde bereits bei den ersten Zugversuchen mit untersucht. 


\section{Zugversuche:}

Zur Absicherung der Versuche wurden vor den Fahrversuchen an den Materialpaarungen der o.g. Fertigteilstapel die Reibwerte nochmals ermittelt.

\section{Bremsversuche:}

Für die Bremsversuche erfolgte die Sicherung der beiden Stapel auf dem Auflieger durch jeweils drei Zurrgurte. Die notwendigen Sicherungskräfte und die daraus abzuleitenden Vorspannungen der Zurrgurte wurde mit den Gesamtgewichten der Stapel und den Reibwerten aus den ersten Versuchen errechnet und an den gesicherten Stapeln überprüft.

Der Auflieger mit den beiden Fertigteilstapeln wurde an eine Sattel-Zugmaschine der DEKRA angekoppelt und mit mehreren triaxialen Beschleunigungsaufnehmern versehen, die bei den Bremsversuchen die auftretenden horizontalen Bremsbeschleunigungen dokumentieren sollten. Das Gesamtgewicht des Sattelzuges betrug 30.550 $\mathrm{kg}$

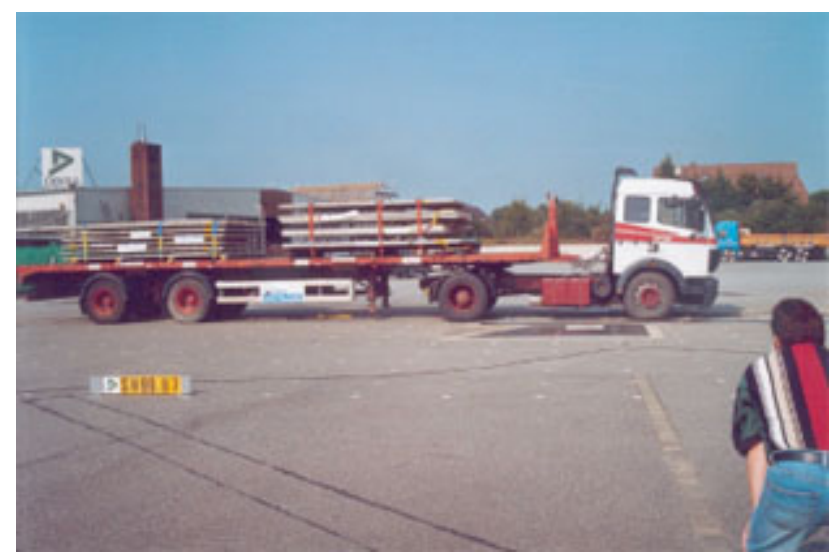

Abbildung 4: Bremsversuch

Um die Auswirkungen unterschiedlicher Verzögerungen auf die Ladung feststellen zu können, wurde der Sattelzug zunächst auf trockener Fahrbahn mit der Betriebsbremse verzögert. Anschließend wurden drei Bremsversuche durchgeführt, bei denen als maximaler Belastungsfall $\mathrm{a}=0,81 \mathrm{~g}$ erreicht wurden. Abschließend wurde ein Versuch durchgeführt, bei dem jeweils der mittlere Zurrgurt der Ladungssicherung entfernt wurde. Insgesamt zeigten die Bremsversuche, dass die durch die Zugversuche ermittelten Reibwerte gut dazu geeignet waren, die Ladungssicherung entsprechend der Maßgabe der VDI-Richtlinie 2700 ff. auszulegen.

\section{Weiteres Vorgehen}

Die Ergebnisse der Zugversuche und die guten Ergebnisse der Bremsversuche im DEKRA-Crashzentrum in Neumünster ermöglichten eine zuverlässige Festlegung von Reibzahlen als erforderliche Grundlage für die Auswahl der Ladungssicherungsmaßnahmen. Es werden vom BDB z. Zt. Empfehlungen für die Auslegung der Ladungssicherungsmaßnahmen für Betonfertigteile erarbeitet.

Neben den Empfehlungen soll eine Tabelle mit Angaben zu Reibwerten für verschiedene Materialpaarungen von Beton gegen z.B. Holz oder Stahl dargestellt werden, die als Grundlage für die Berechnung der Sicherungskräfte beim Niederzurren dienen soll.

Die Erarbeitung und Festlegung der nachfolgenden Reibwerttabelle wurde basierend auf allen Messergebnissen der Zugversuche durchgeführt. 
Tabelle 1: Gleitreibzahlen für trockene und nasse Ladeflächen.

\begin{tabular}{|l|r|}
\hline $\begin{array}{l}\text { Materialp aarung } \\
\text { Elementdecke auf Stahlarmierung }\end{array}$ & $\begin{array}{r}\text { Gleitreibzahl } \boldsymbol{~} \\
0,60\end{array}$ \\
\hline Elementdecke /Elementwand auf Holz & $\begin{array}{r}\text { gebrauct } 0,50 \\
\text { neuwertig } 0,40\end{array}$ \\
\hline Elementwand auf Elementwand & 0,55 \\
\hline Stahlrahmen auf Holzladıfläche & 0,40 \\
Holzbalken auf Holzladeflätie & 0,50 \\
\hline & \\
\hline
\end{tabular}

Diese Tabelle und die vom BDB ausgearbeiteten Empfehlungen zur Ladungssicherung von Betonfertigteilen sollen in die Novellierung der VDI-Richtlinie 2700 einfließen und als eigenständiges Blatt innerhalb der Richtlinien-Reihe erscheinen. 